1989, 38, 1

УдК 517.5

J. LIPPUS

\title{
ON THE PRESERVATION OF CLASSES OF FUNCTIONS
}

\author{
(Presented by G. Vainikko)
}

If $X$ and $Y$ are two classes of $2 \pi$-periodic functions, we say that a twoway infinite series of complex numbers $\lambda=\left\{\lambda_{k}\right\} \quad(-\infty \leqslant k \leqslant \infty)$ is a multiplier from $X$ into $Y$, and we write $\lambda \in(X, Y)$ if whenever

$$
\sum_{k=-\infty}^{\infty} c_{k} \mathrm{e}^{\mathrm{i} h x}
$$

is the Fourier series of a function $f$ in $X$, the series

$$
\sum_{k=-\infty}^{\infty} \lambda_{k} c_{k} \mathrm{e}^{\mathrm{i} k x}
$$

is the Fourier series of a function $f_{\lambda}$ in $Y$. Let $C$ denote the class of $2 \pi$ periodic continuous functions with the norm

$$
\|f\|_{C}=\max _{0 \leqslant x \leqslant 2 \pi}|f(x)|,
$$

and $L$ the class of $2 \pi$-periodic integrable functions with the norm

$$
\|f\|_{L}=\frac{1}{2 \pi} \int_{0}^{2 \pi}|f(x)| \mathrm{d} x .
$$

Let $\omega(\delta)$ be a given modulus of continuity and let $C_{\omega}$ denote the class of continuous functions, for the moduli of continuity

$$
\omega(f, \delta)=\sup _{|h| \leqslant \delta}\|f(\cdot+h)-f(\cdot)\|_{c}
$$

of which we have

$$
\omega(f, \delta)=O(\omega(\delta)) .
$$

It is well known (see e.g. $\left.{ }^{1}\right]$, p. 176) that a necessary and sufficient condition for a sequence $\lambda$ to be of the type $(C, C)$ is that

$$
\sum_{k=-\infty}^{\infty} \lambda_{k} \mathrm{e}^{\mathrm{i} k x}
$$

be a Fourier-Stieltjes series. For the type $\left(C_{\omega}, C_{\omega}\right)$ this condition while remaining sufficient ceases to be necessary. For the Lipschitz classes A. Zygmund ${ }^{[2]}$ showed that a necessary and sufficient condition for $\lambda$ to be of the type $\left(C_{\omega}, C_{\omega}\right)$ with $\omega(\delta)=\delta^{\alpha}(0<\alpha<1)$ is that the indefinite integral of the series (3)

$$
\mathfrak{L}(x)=\sum_{k=-\infty}^{\infty} \frac{\lambda_{k}}{\mathrm{i} k} \mathrm{e}^{\mathrm{i} k x}
$$

should belong to the Zygmund class in the integral metrics $L_{*}$, i. e. 


$$
\omega_{2}(\hat{f}, \delta)_{L}=\sup _{|h| \leqslant \delta}\|\dot{f}(\cdot+2 h)+\dot{f}(\cdot)-2 \dot{f}(\cdot+h)\|_{L}=\dot{O}(\delta) .
$$

Here the dash indicates that the term with the zero index is absent.

In the present paper we extend this result to a somewhat wider class of moduli of continuity, satisfying the condition

$$
\int_{0}^{\delta} \frac{\omega(t)}{t} \mathrm{~d} t+\delta \int_{\delta}^{2 \pi} \frac{\omega(t)}{t^{2}} \mathrm{~d} t=O(\omega(\delta))
$$

(see e. g. $\left[{ }^{3}\right]$ or $\left[{ }^{4}\right]$, p. 420 ). We also show that this extension is maximal in its terms.

Throughout this paper we suppose that $\omega(\delta) / \delta \rightarrow \infty(\delta \rightarrow 0+)$.

Theorem 1. A necessary condition for the sequence $\lambda=\left\{\lambda_{k}\right\}$ to be of the type $\left(C_{\omega}, C_{\omega}\right)$ is that $\mathbb{R}$ should belong to the class $L_{*}$. If the modulus of continuity $\omega(\delta)$ is such that (5) holds, then this condition is also sufficient.

P r o of. Let $X$ be either $C$ or $L$. Let $P_{n}$ denote the set of all trigonometric polynomials of an order not higher than $n$, For $f \in X$ let

$$
E_{n}(f)_{X}=\inf _{T \in P_{n}}\|f-T\|_{X}
$$

denote the best approximation of the function $f$. Let $f * g$ denote the convolution

$$
(f * g)(x)=\frac{1}{2 \pi} \int_{0}^{2 \pi} f(x-t) g(t) \mathrm{d} t .
$$

$v_{n} f$ the de la Vallée Poussin means of the series (1)

$$
v_{n} f=(1 / n)\left(s_{n} f+s_{n+1} f+\ldots+s_{2 n-1} f\right)
$$

and $v_{n}$ the corresponding kernel $v_{n} f=v_{n} * f, v_{n}(x)=2 K_{2 n-1}(x)-K_{n-1}(x)$ where

$$
K_{n}(x)=\sum_{k=-n}^{n}(1-|k| /(n+1)) \mathrm{e}^{\mathrm{i} k x}
$$

is the Fejér kernel. For any complex number let $z$ define the function $\overline{\operatorname{sign}} z=\bar{z} /|z|$ for nonzero $z, \overline{\operatorname{sign}} z=0$ if $z=0$.

Suppose $\mathcal{Q} \in L_{*}$ and let $F_{\lambda}$ denote the indefinite integral of $f_{\lambda}$. Then we may write

$$
E_{n}\left(F_{\lambda}\right)_{c}=E_{n}(f * \Omega)_{c} \leqslant E_{n}(f)_{C} E_{n}(\Omega)_{L} .
$$

Applying Jackson's theorem twice we see that the first term on the right is $O(\omega(1 / n))$ and the second term is $O(1 / n)$. Thus

$$
E_{n}\left(F_{\lambda}\right)_{C}=O((1 / n) \omega(1 / n)) .
$$

In view of (5) (see e.g. $\left[{ }^{3}\right]$ or $\left[{ }^{4}\right]$, p. 423 ) this is equivalent to

$$
\omega(f \lambda, \delta)=O(\omega(\delta)) \text {, }
$$

i. e. $f_{\lambda}$ is in $C_{\omega}$. This proves the sufficiency part of the theorem. To prove the necessity part suppose that $\Omega \equiv L_{*}$. In that case there exists a sequence of indices $\{n(k)\}$ such that

$$
\left\|v_{2 n(k)} \mathfrak{Q}-v_{n(k)} \mathfrak{R}\right\|_{L} \geqslant \frac{2^{k}}{n_{k}} .
$$

If it were not true, i. e. if $\left\|v_{2 n} \mathfrak{\Omega}-v_{n} \mathfrak{\Omega}\right\|_{L}=O(1 / n)$ we could write for any $n$ 


$$
\left\|\mathfrak{I}-v_{n} \mathfrak{\Omega}\right\|_{L}=\left\|\sum_{k=0}^{\infty}\left\{{v^{k+1}} \mathfrak{\Omega}-v_{2^{k} n} \Omega\right\}\right\|_{L} \leqslant \sum_{k=0}^{\infty} O\left(\frac{1}{2^{k} n}\right)=O(1 / n),
$$

which contradicts the presumption that $\Omega \equiv L_{*}$.

Moreover, since we have presumed that $\omega(\delta) / \delta \rightarrow \infty(\delta \rightarrow 0+)$, we may suppose that the sequence $\{n(k)\}$ satisfies the conditions

$$
\begin{aligned}
& n(k) \geqslant 8 n(k-1), \\
& \omega(1 / n(k)) \leqslant(1 / 2) \omega(1 / n(k-1)), \\
& n(k) \cdot \omega(1 / n(k)) \geqslant \sum_{l=1}^{k-1} n(l) \cdot \omega(1 / n(l)) .
\end{aligned}
$$

Consider the functions $(k=1,2, \ldots)$

$$
G_{k}(x)=\overline{\operatorname{sign}}\left\{v_{2 n(k)} \mathfrak{Q}(-x)-v_{n(k)} \mathfrak{Q}(-x)\right\}
$$

and

$$
\varphi_{k}(x)=G_{k}(x) *\left\{v_{2 n(k)}(x)-v_{n(k)}(x)\right\} .
$$

The functions $\varphi_{k}$ are trigonometric polynomials of the order $4 n(k)$. Since $\left\|v_{n}\right\|_{L}=O(1)$ (see e.g. $\left.{ }^{1}\right]$, p. 88 ) we have by Young's inequality that $\left\|\varphi_{k}\right\|_{C}=O(1)$. Define a function $f$ by the series

$$
f(x)=\sum_{k=1}^{\infty} \omega(1 / n(k)) \varphi_{k}(x) .
$$

In view of (8) this series converges uniformly, hence $f \in C$. Let us estimate the modulus of continuity of $f$. Let $1 / n(k+1) \leqslant \delta \leqslant 1 / n(k)$. We have

$$
\|f(\cdot+\delta)-f(\cdot)\|_{c} \leqslant
$$

$$
\leqslant\left\|\sum_{l=1}^{k} \omega(1 / n(l))\left\{\varphi_{l}(\cdot+\delta)-\varphi_{l}(\cdot)\right\}\right\|_{C}+2 \sum_{l=k+1}^{\infty} \omega(1 / n(l))\left\|\varphi_{l}\right\|_{C} .
$$

As $\left\|\varphi_{l}\right\|_{C}=O(1)$ the last sum is $O(\omega(\delta))$. The first sum we estimate applying Bernstein's inequality to the polynomials $\varphi_{k}$ and using (9)

$$
\begin{gathered}
\sum_{l=1}^{k} \omega(1 / n(l))\left\|\varphi_{l}(\cdot+\delta)-\varphi_{l}(\cdot)\right\|_{C}=O\left(\sum_{l=1}^{k} n(l) \omega(1 / n(l)) \delta\right)= \\
=O(\delta n(k+1) \omega(1 / n(k+1))) .
\end{gathered}
$$

Since any modulus of continuity has the property $\omega\left(t_{1}\right) / t_{1} \leqslant 2 \omega\left(t_{2}\right) / t_{2}$ for $t_{2} \leqslant t_{1}$ we conclude that the first sum is also $O(\omega(\delta))$, hence $f \in C_{\omega}$.

Let $m(k)=[n(k) / 2]$, where [x] denotes the entire part of $x$. Consider the difference

$$
v_{4 n(k)} F_{\lambda}-v_{m(k)} F_{\lambda} .
$$

If $f_{\lambda} \in C_{\omega}$ then we should have by Jackson's theorem (see e. g. $\left[{ }^{4}\right]$, p. 275) $\left\|v_{4 n(k)} F_{\lambda}-v_{m(k)} F_{\lambda}\right\|_{c} \leqslant 4\left\{E_{4 n(k)}\left(F_{\lambda}\right)_{c}+E_{m(k)}\left(F_{\lambda}\right)_{c}\right\}=$

$$
=O\left(\frac{1}{n(k)} \omega\left(\frac{1}{n(k)}\right)\right) \text {. }
$$

On the other hand, we have constructed $f$ in such a way that by virtue of $(7)$

$$
v_{4 n(k)} F_{\lambda}-v_{m(k)} F_{\lambda}=\left\{v_{4 n(k)} f-v_{m(k)} f\right\} * \mathfrak{X}=\omega(1 / n(k))\left\{\varphi_{k} * \mathfrak{X}\right\} .
$$

Since $\varphi_{k}$ in its turn is a convolution we may write

$$
\varphi_{k} * \mathfrak{R}=\overline{\operatorname{sign}}\left\{v_{2 n(k)} \mathfrak{R}(-x)-v_{n(k)} \mathfrak{R}(-x)\right\} *\left\{v_{2 n(k)} \mathfrak{L}(x)-v_{n(k)} \mathfrak{R}(x)\right\} .
$$


Applying (6) obtain the estimate

$$
\left\|\varphi_{k} * \Omega\right\|_{C} \geqslant\left(\varphi_{k} * \Omega\right)(0) \geqslant
$$

$\geqslant \frac{1}{2 \pi} \int_{0}^{2 \pi} \overline{\operatorname{sign}}\left\{v_{2 n(k)} \mathfrak{Q}(-t)-v_{n(k)} \mathfrak{R}(-t)\right\} *\left\{v_{2 n(k)} \mathfrak{Q}(-t)-v_{n(k)} \mathfrak{Q}(-t)\right\} \mathrm{d} t=$

$$
=\left\|v_{2 n(k)} \mathfrak{L}-v_{n(k)} \mathfrak{R}\right\|_{L} \geqslant 2^{k} / n(k) .
$$

Thus

$$
\left\|v_{4 n(k)} F_{\lambda}-v_{m(k)} F_{\lambda}\right\|_{C} \geqslant 2^{h_{\omega}}(1 / n(k)) / n(k),
$$

which contradicts $(10)$. Hence $f_{\lambda} \equiv C_{\omega}$. This concludes the proof of Theorem 1.

To show that without condition (5) theorem 1 ceases to be true let us prove the following

Theorem 2. Let the sequence $\lambda=\left\{\lambda_{k}\right\}$ be such that $\Omega \in L_{*}$ but (3) is not a Fourier-Stieltjes series. Then there exists a modulus of continuity $\omega(\delta)$ not satisfying the condition (5), and a function $f \in C_{\omega}$ such that $f_{\lambda}$ does not belong to $C_{\omega}$.

To prove the theorem we shall use some K. I. Oskolkov's results $\left(\left[{ }^{5}\right],\left[{ }^{6}\right]\right)$.

Lemma $\left(\left[{ }^{5}\right]\right.$, lemma 2$)$. For any sequence $\left\{\delta_{k}\right\} \quad(0 \leqslant k \leqslant \infty)$ with the properties

$$
\delta_{0}=\pi, \quad \delta_{k}>0, \quad 4 \delta_{k+1} / \delta_{k} \leqslant 1 \quad(k=0,1,2, \ldots),
$$

there exists a modulus of continuity $\omega(\delta)$ such that

$$
\delta_{k+1}=\min \left\{\delta: \max \left(\frac{\omega(\delta)}{\omega\left(\delta_{k}\right)}, \frac{\delta \omega\left(\delta_{k}\right)}{\delta_{k} \omega(\delta)}\right)=\frac{1}{2}\right\} \quad(k \geqslant 0)
$$

and

$$
\frac{1}{C} \omega(\delta) \leqslant \sum_{k=0}^{\infty} \omega\left(\delta_{k}\right) \min \left\{1, \delta / \delta_{k}\right\} \leqslant C \omega(\delta) \quad(\delta>0) ;
$$

$\omega(\varepsilon)$ may be selected such that

$$
\omega\left(\delta_{k+1}\right)=(1 / 2) \omega\left(\delta_{k}\right) .
$$

Moreover, the condition

$$
\delta_{k} / \delta_{k+1}=O(1) \quad(k \rightarrow \infty)
$$

is equivalent to (5) ([5], remark 2).

Let $v_{n} \Lambda$ denote the de la Vallée Poussin means of the series (3). Since the latter is not a Fourier-Stieltjes series we have

$$
\underset{n}{\limsup }\left\|v_{n} \Lambda\right\|_{L}=\infty
$$

(see e. g. $\left[{ }^{1}\right]$, p. 137). Hence there exists a sequence of indices $\{n(k)\}$ $(k=1,2, \ldots)$ such that

$$
n(k+1) / n(k) \rightarrow \infty \quad(k \rightarrow \infty)
$$

and

$$
\left\|v_{n(k+1)} \Lambda-v_{n(k)} \Lambda\right\|_{L} \geqslant k .
$$

We may suppose that $n(1)>4 \pi$ and $n(k+1)>4 n(k)$. Consider the sequence $\left\{\delta_{k}\right\}$, where

$$
\delta_{0}=\pi, \quad \delta_{k}=1 / n(k) \quad(k=1,2, \ldots) .
$$


Let $\omega(\delta)$ be the corresponding modulus of continuity according to the lemma. In view of (13) observe that (5) does not hold.

Let

$$
G_{k}(x)=\overline{\operatorname{sign}}\left\{v_{n(k+1)} \Lambda(-x)-v_{n(k)} \Lambda(-x)\right\}
$$

and

$$
\varphi_{k}(x)=G_{k}(x) *\left\{v_{n(k+1)}(x)-v_{n(k)}(x)\right\} .
$$

These functions are trigonometric polynomials of the order $2 n(k+1)$ orthogonal to trigonometric polynomials of an order less than $n(k)$. We also have $\left\|\varphi_{k}\right\|_{C}=O(1)$. Let

$$
f(x)=\sum_{k=1}^{\infty} \omega\left(\delta_{k}\right) \varphi_{k}(x)
$$

By (12) this series converges uniformly, hence $f \in C$. To estimate the modulus of continuity of $f$ notice that by Bernstein's inequality we have

$$
\omega\left(\varphi_{k}, \delta\right)=O(\min \{1, n(k+1) \delta\})=O\left(\min \left\{1, \delta / \delta_{k}\right\}\right) .
$$

Applying (12) and (11) we see that $f \in C_{\omega}$.

Next prove that $f_{\lambda} \equiv C_{\omega}$. If $f$ is to belong to $C_{\omega}$ we should have $(m(k)=[n(k) / 2])$

$$
\begin{gathered}
\left\|v_{2 n(k+1)} f_{\lambda}-v_{m(k)} f_{\lambda}\right\|_{C} \leqslant 4\left(E_{2 n(k+1)}\left(f_{\lambda}\right)_{C}+E_{m(k)}\left(f_{\lambda}\right)_{C}\right)= \\
1=O(\omega(1 / n(k)) .
\end{gathered}
$$

On the other hand we have defined $\varphi_{k}$ so that

$$
\begin{gathered}
v_{2 n(k+1)} f_{\lambda}-v_{m(k)} f_{\lambda}= \\
=\left\{\omega\left(\delta_{k-1}\right) \varphi_{k-1}+\omega\left(\delta_{k}\right) \varphi_{k}+\omega\left(\delta_{k+1}\right) \varphi_{k+1}\right\} *\left\{v_{2 n(k+1)} \Lambda-v_{m(k)} \Lambda\right\} .
\end{gathered}
$$

Considering that all terms in the convolution are trigonometric polynomials of appropriately chosen order we get

$$
\begin{gathered}
v_{2 n(k+1)} f_{\lambda}-v_{m(k)} f_{\lambda}=\omega\left(\delta_{k}\right)\left(\varphi_{k}\right)_{\lambda}+\omega\left(\delta_{k-1}\right) G_{k-1} *\left\{v_{n(k)} \Lambda-v_{m(k)} \Lambda\right\}+ \\
+\omega\left(\delta_{k+1}\right) G_{k+1} *\left\{v_{2 n(k+1)} \Lambda-v_{n(k+1)} \Lambda\right\} .
\end{gathered}
$$

Since $\left\|G_{k}\right\|_{c} \leqslant 1$ the application of Young's inequality gives us

$$
\begin{gathered}
\left\|v_{2 n(k+1)} f_{\lambda}-v_{m(k)} f_{\lambda}\right\|_{C} \geqslant \omega\left(\delta_{k}\right)\left(\varphi_{k}\right)_{\lambda}(0)-\omega\left(\delta_{k-1}\right)\left\|v_{n(k)} \Lambda-v_{m(k)} \Lambda\right\|_{L}- \\
-\omega\left(\delta_{k+1}\right)\left\|v_{2 n(k+1)} \Lambda-v_{n(k+1)} \Lambda\right\|_{L} .
\end{gathered}
$$

As $\Omega \in L_{*}$ we have by Bernstein's inequality for arbitrary $n$ and $m$ $(n>m)$

$$
\begin{aligned}
& \left\|v_{n} \Lambda-v_{m} \Lambda\right\|_{L} \leqslant 2 n\left\|v_{n} \Omega-v_{m} \Omega\right\|_{L} \leqslant \\
& \leqslant n O\left(E_{n}(\Omega)_{L}+E_{m}(\Omega)_{L}\right)=n O(1 / n+1 / m) .
\end{aligned}
$$

If $n / m=O(1)$ this yields $\left\|v_{n} \Lambda-v_{m} \Lambda\right\|_{L}=O(1)$. Noting that $m(k)=$ $=[n(k) / 2]$ and (12) holds we deduce from (16)

$$
\left\|v_{2 n(k+1)} f_{\lambda}-v_{m(k)} f_{\lambda}\right\|_{C} \geqslant \omega\left(\delta_{k}\right)\left(\varphi_{k}\right)_{\lambda}(0)+O\left(\omega\left(\delta_{k}\right)\right) .
$$

Using the construction of $\varphi_{k}$ we obtain by virtue of (14)

$$
\begin{gathered}
\left(\varphi_{k}\right)_{\lambda}(0)=\frac{1}{2 \pi} \int_{0}^{2 \pi} \overline{\operatorname{sign}}\left\{v_{n(k+1)} \Lambda(-t)-v_{n(k)} \Lambda(-t)\right\} * \\
*\left\{v_{n(k+1)} \Lambda(-t)-v_{n(k)} \Lambda(-t)\right\} \mathrm{d} t=\left\|v_{n(k+1)} \Lambda-v_{n(k)} \Lambda\right\|_{L} \geqslant k .
\end{gathered}
$$


Thus

$$
\left\|v_{2 n(k+1)} f_{\lambda}-v_{m(k)} f_{\lambda}\right\|_{C} \geqslant \omega\left(\delta_{k}\right) k+O\left(\omega\left(\delta_{k}\right)\right)
$$

which contradicts (15). Therefore $f_{\lambda}$ does not belong to $C_{\omega}$.

Remark. Analogous statements are also true for the class $\left(L_{\omega}, L_{\omega}\right)$. In that case while constructing the counterexamples we may suppose $G_{k} \equiv 1(k=1,2, \ldots)$. The rest of the proof remains essentially the same.

\section{REFERENCES}

1. Zygmund, A. Trigonometric Series. Cambridge, Cambridge University Press, 1959, 1.

2. Żygmund, A. // J. Math. Mech., 1959, 8, № 6, 889-895.

3. Бари Н. К., Стечкин С. Б. // Тр. Моск. Мат. об-ва, 1956, 5, 434-522.

4. Тиман А. Ф. Теория приближения функций действительного переменного. М., Физматгиз, 1960.

5. Осколков К. Н. // Тр. МИАН СССР, 1983, 164, 124-135.

6. Осколков К. Н. // Мат. сб., 1977, 103, № 4, 536-589.

Academy of Sciences of the Estonian SSR, Institute of Cybernetics

Received Dec. 1,1987

\section{J. LIPPUS}

\section{FUNKTSIOONIDE KLASSIDE SÄILITAMISEST}

Artiklis on leitud tarvilikud ja piisavad tingimused selleks, et kompleksarvude jada $\lambda=\left\{\lambda_{k}\right\}$ oleks $\left(C_{\omega}, C_{\omega}\right)$-tüüpi multiplikaatoriks eeldusel, et pidevuse moodul $\omega(\delta)$ rahuldab tingimust (5). On näidatud, et kui hinnang (5) ei ole täidetud, siis teoreem enam ei kehti.

\section{ю. липпус}

\section{О СОХРАНЕНИИ КЛАССОВ ФУНКЦИИ}

Находятся необходимые и достаточные условия для того, чтобы последовательность комплексных чисел $\lambda=\left\{\lambda_{k}\right\}$ являлась мультипликатором типа $\left(C_{\omega}, C_{\omega}\right)$ в предположении, что модуль непрерывности $\omega(\delta)$ удовлетворяет условию (5). Также поқазывается, что без условия (5) теорема перестает быть верной. 